\title{
Modified Facial Artery Musculomucosal Flap for Reconstruction of Posterior Skull Base Defects
}

\author{
Liyue Xie ${ }^{1,2}$ Philippe Lavigne ${ }^{1,2}$ François Lavigne \\ ${ }^{1}$ Department of Otolaryngology-Head and Neck Surgery, Université \\ de Montréal, CHUM-Hôpital Notre-Dame, Montreal, Quebec, Canada \\ 2 Department of Otolaryngology-Head and Neck Surgery, Université \\ de Montréal, Hôpital Maisonneuve-Rosemont, Montreal, Quebec, \\ Canada
}

Tareck Ayad ${ }^{1,2}$

J Neurol Surg Rep 2016;77:e98-e101.

\begin{abstract}
Address for correspondence Philippe Lavigne, MD, Department of Otolaryngology-Head and Neck Surgery, Université de Montréal, CHUM-Hôpital Notre-Dame, 1560 Sherbrooke Street East, Montreal, Quebec, Canada, H2L 4M1

(e-mail: philippe.lavigne@umontreal.ca).
\end{abstract}

\begin{abstract}
Keywords

- skull base

- reconstruction

- facial artery musculomucosal flap

- chordoma

Objectives The superiorly pedicled facial artery musculomucosal (FAMM) flap has been successfully used for reconstruction of head and neck defects since 1992. Common sites of defects include the oral cavity and oropharynx. This article presents a clinical case in which we have successfully used a newly developed modification of the FAMM flap for bulky nasopharyngeal and skull base reconstruction.

Results Our patient is a 71-year-old man who presented with a large parapharyngeal and clival chordoma. After tumor removal through combined endoscopic and cervical approach, the internal carotid artery (ICA) in the nasopharyngeal portion was left exposed. A modified superiorly based FAMM flap measuring up to $10 \mathrm{~cm}$ in length and $2.5 \mathrm{~cm}$ in width was successfully harvested and used to completely cover the defect and the ICA. The flap survived local radiation therapy at the long-term follow-up.

Conclusion We have developed a new modification of the FAMM flap, using the fascia of the masseter muscle. This is the first reported case in the literature using a modified FAMM flap for the reconstruction of nasopharyngeal and skull base defect.
\end{abstract}

\section{Introduction}

The facial artery musculomucosal (FAMM) flap was first described by Pribaz et al in $1992 .{ }^{1}$ It consists of the mucosa and the submucosa of the cheek and a portion of the buccinator muscle. It can be pedicled inferiorly on the facial artery and superiorly on the angular artery, depending on the site of the defect that needs reconstruction. The superiorly based FAMM flap was successfully used in the reconstruction of the palate, upper vestibule, nasal cavity, and orbit. ${ }^{2}$ Recent research focused on developing modifications to expand the indications of this flap. ${ }^{3,4}$ Among these, Xie et al $^{5}$ have developed a modification of the FAMM flap in cadavers that increased its length by adding an extension using the fascia of the masseter for skull base reconstruction. They demonstrated the feasibility of using this wellvascularized flap for a tension-free coverage of key areas of the skull base.

received

July 15, 2015

accepted after revision

December 30, 2015 10.1055/s-0036-1579630. ISSN 2193-6358.
The details of the surgical technique are well described and illustrated in their study. ${ }^{5}$ In summary, this modification required a submandibular incision for dissection and ligation of the cervical facial artery. Then, the buccal and marginal branches of the facial nerve are carefully dissected from the parotidomasseteric fascia to prevent a lower facial paralysis. Care should be taken to leave the masseteric fascia in continuity with the facial artery for proper vascular perfusion of the extension flap. The fascia and the facial artery are then transferred intraorally through a blunt dissection anterior to the masseter muscle. The remaining steps in the harvest of the FAMM flap are achieved according to the standard technique. ${ }^{1}$

The use of the FAMM flap in posterior skull base or nasopharynx reconstruction has never been reported in a living patient. We report the first case of skull base reconstruction using a superiorly pedicled FAMM flap modified with an extension to the masseteric fascia and muscle in a
License terms

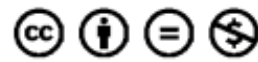

(c) 2016 Georg Thieme Verlag KG Stuttgart · New York 


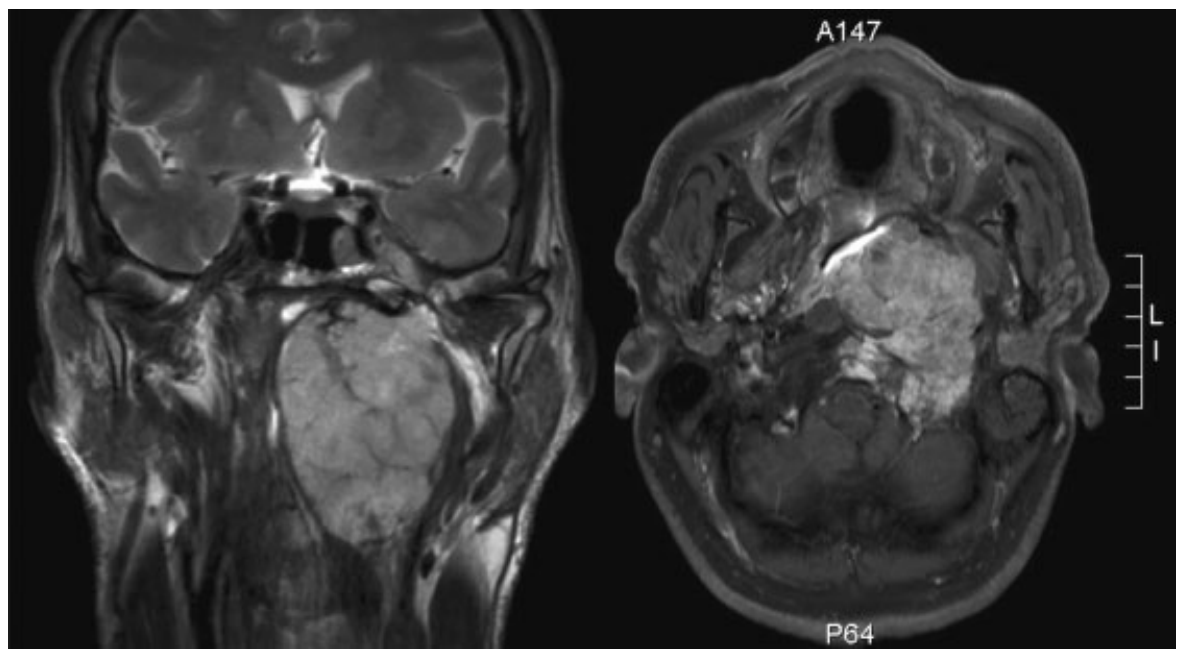

Fig. 1 Preoperative MRI axial and coronal views in T2W-TSE showing voluminous tumor mainly located in the left parapharyngeal space with superior invasion of the clivus, lateral process of $\mathrm{C} 1$, and the left occipital condyle.

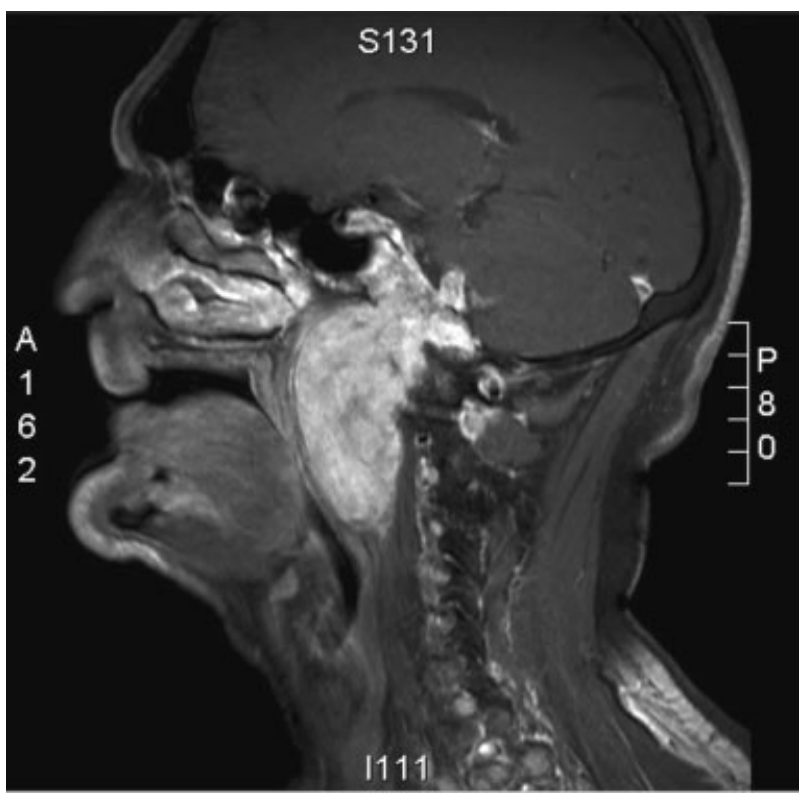

Fig. 2 Preoperative MRI sagittal T1W-TSE C+ showing the craniocaudal extension of tumor. living patient. The defect involved the exposure of the nasopharyngeal and petrous portions of the internal carotid after an extensive surgical resection of a chordoma.

\section{Case Report}

Our patient is a 71-year-old man who presented at our clinic with odynophagia, dysphagia, atrophy of the left hemi tongue, and diplopia. His past medical history included dyslipidemia, hypertension, diabetes, and myocardial infarct. Imaging investigations showed the presence of a voluminous lesion $(4.8 \times 6.5 \times 8.7 \mathrm{~cm})$ in the left parapharyngeal space with superior invasion of the clivus, lateral process of $\mathrm{C} 1$, and the left occipital condyle (-Figs. 1 and $\mathbf{2}$ ). A biopsy confirmed the diagnosis of a chordoma (-Fig. 3 ).

He first underwent a fusion of the occipital bone to $\mathrm{C} 5$ for cervical instability. Tumor removal required an endoscopic transnasal approach combined with an open cervical approach for excision of the left parapharyngeal extension. Because adjuvant radiation therapy was planned for this patient, the defect would best be reconstructed using a robust vascularized flap.

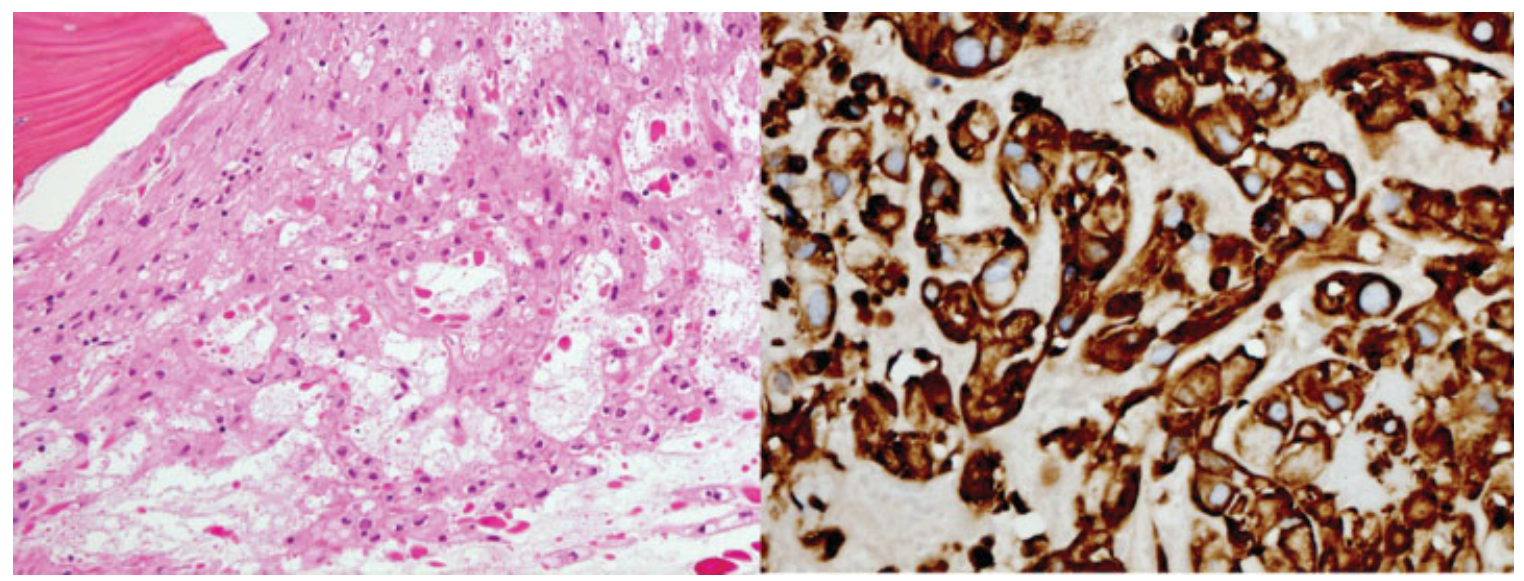

Fig. 3 Histologic sections demonstrating cords and lobules of large cells, with small, bland nuclei and abundant cytoplasm, eosinophilic or vacuolated, in a loose, myxoid stroma. These cells are positive for the epithelial markers (CK AE1/3, CK8-18, EMA), vimentin and brachyury; all other markers were negative (S100, RCC, CK5/6, CEA, MOC31). 
We harvested an extended retrograde FAMM flap measuring $10 \mathrm{~cm}$ in length and $2.5 \mathrm{~cm}$ in width (-Fig. 4). The flap was transferred through a Caldwell-Luc fenestration to the maxillary sinus (- Fig. 5A). An endoscopic maxillary antrostomy was performed and allowed the flap to reach the nasopharynx by passing through the maxillary sinus (-Fig. 5B). Once in place, the flap completely covered the petrous portion of the exposed internal carotid and the extension island flap covered the retrostyloid parapharyngeal space. The flap was held in place with fibrin sealant topical and petroleum gauze nasal packing to reduce the risk of retraction from the pulling gravity force.

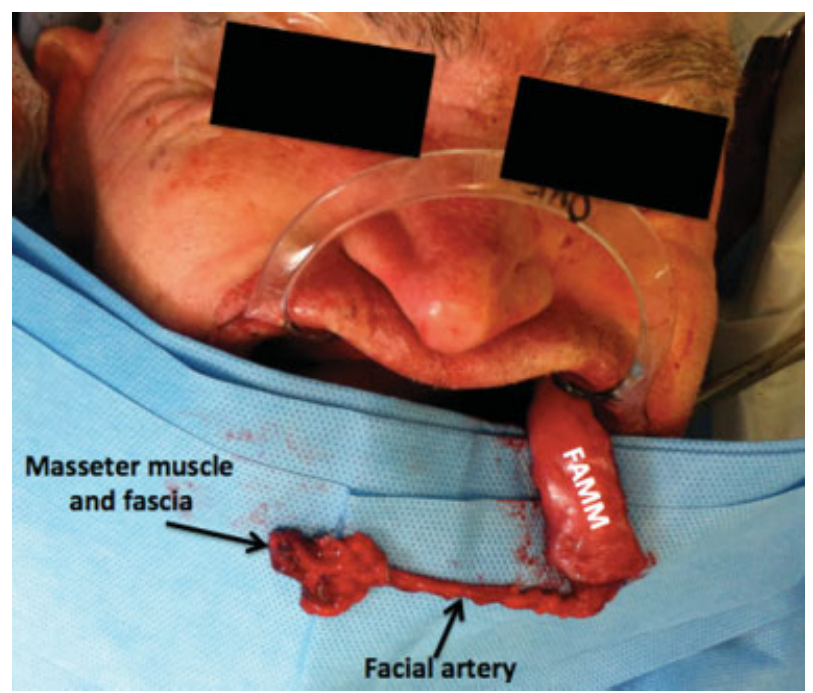

Fig. 4 Perioperative view of the facial artery musculomucosal (FAMM) flap with an island extension to the masseter muscle (FAMME flap).

\section{Discussion}

The workhorse in skull base reconstruction is the vascularized nasoseptal flap as described by Hadad et al in 2006 with the ability to lower cerebrospinal fluid leak rate to $5 \%{ }^{6}$ In this case, the nasoseptal flap or the inferior turbinate flap could not be used because of the extensive invasion of the tumor in the nasal cavity and the posterior septum. We also anticipated that this patient would need postoperative adjuvant radiation therapy based on the size and the extent of the tumor. A pericranial flap would have required a coronal incision leaving a very thin flap

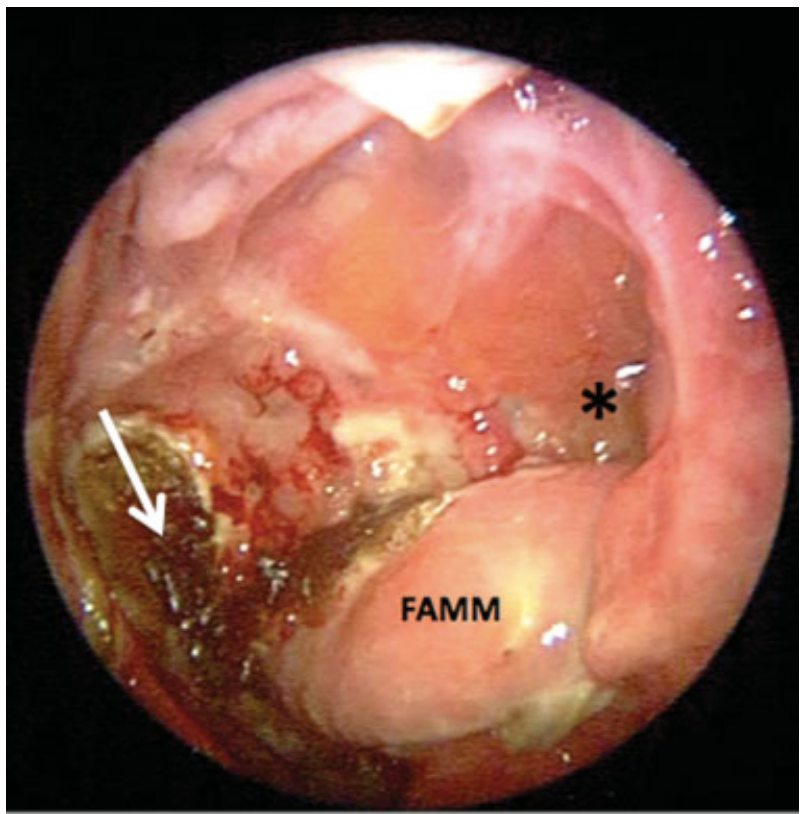

Fig. 6 Endoscopic view of the facial artery musculomucosal (FAMM) flap 2 weeks postoperative. The arrow indicates the fascia of the masseter muscle as the extension flap. ${ }^{*}$ Maxillary sinus.
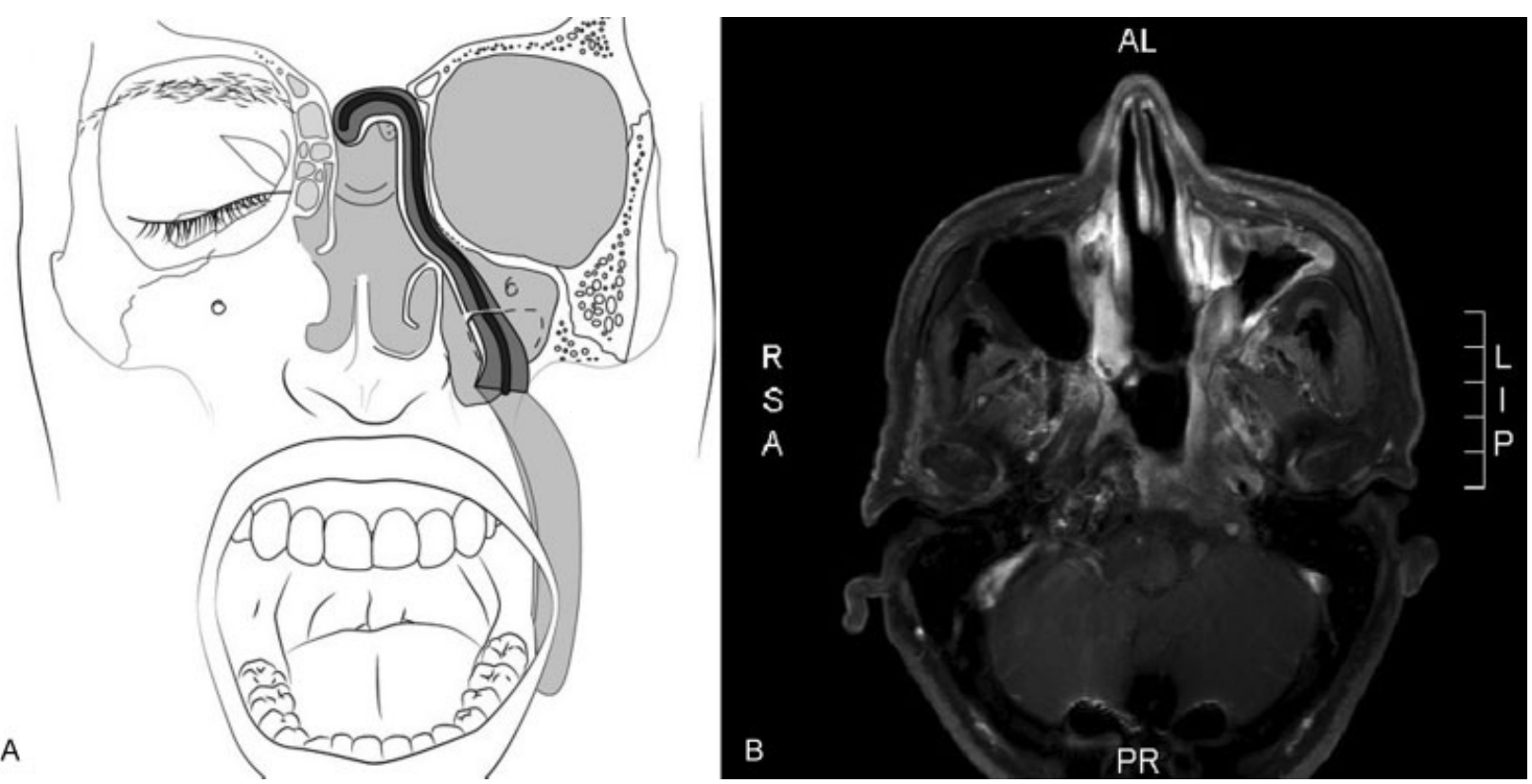

Fig. 5 (A) The transfer of the superiorly pedicled facial artery musculomucosal flap through the maxillary sinus to the skull base. (B) Postoperative MRI T1W-TSE C+ showing the transmaxillary passage of the FAMM flap pedicle. 


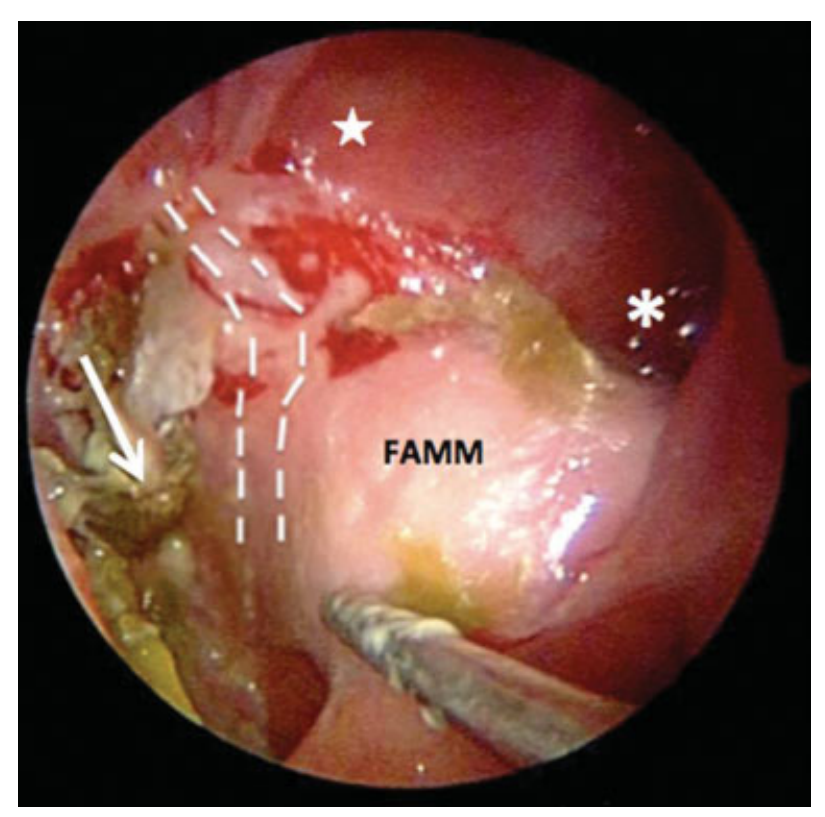

Fig. 7 Endoscopic view of the facial artery musculomucosal (FAMM) flap 2 months postradiation therapy and 5 months postsurgery. The arrow indicates the fascia of the masseter muscle as the extension flap. The dotted line denotes the course of the internal artery covered by the FAMM flap. * The petrous portion of the skull base.* Maxillary sinus.

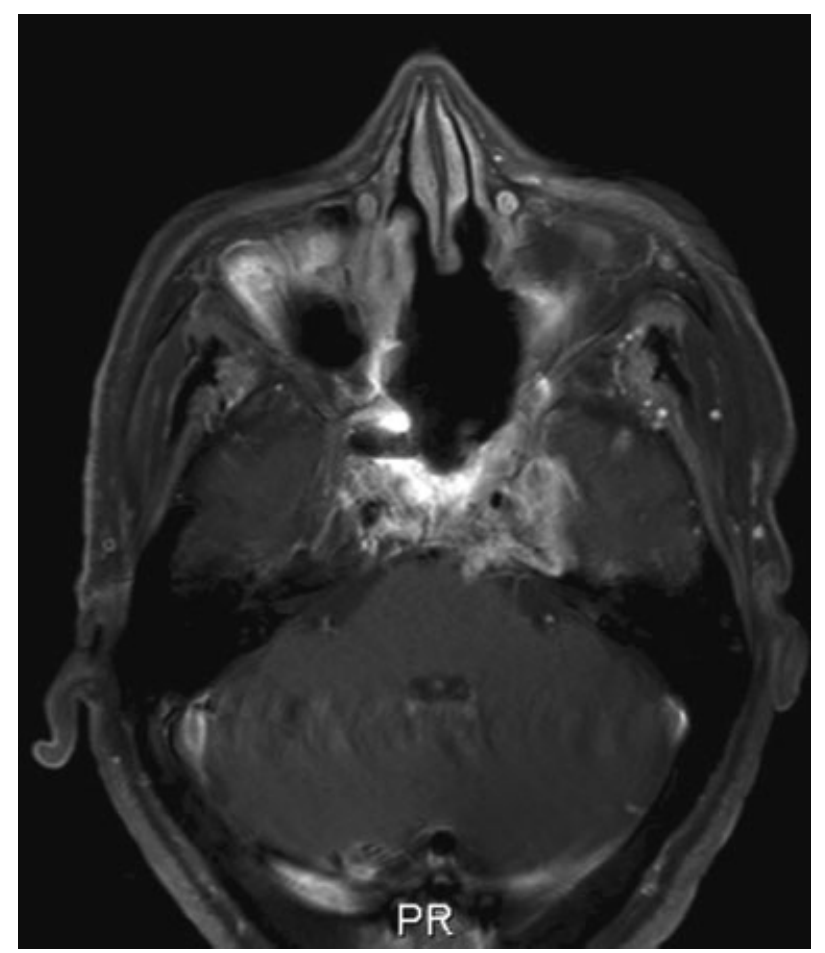

Fig. 8 Postoperative and postradiotherapy axial MRI T1W-TSE C+ showing stability of intracranial and cavernous tumoral residue at 4 years follow-up.

for reconstruction with limited protection for the petrous part of the internal carotid artery (ICA). The use of a free flap was not considered because of the significant comorbidities of this patient.

The FAMM flap was ideal as it is a robust and well-vascularized regional flap. Furthermore, the need of a cervical incision for tumor extirpation also encouraged us to use this flap. Recent studies have focused on developing modifications to expand the indications of this flap by increasing its length. ${ }^{3-5}$ These studies demonstrated the feasibility of using this well-vascularized flap for a tension-free coverage of key areas of the skull base. The details of the surgical technique were well described and illustrated in our previous study. ${ }^{5}$ In this specific case, a portion of the masseter muscle was harvested along with its fascia to obtain a bulkier island extension flap ( - Fig. 4). The benefit of this modification is the location of the island extension being at a certain distance from the distal FAMM flap, which was ideal for our two sites defects (parapharyngeal and petrous carotid) located at a distance from each other.

At 2 weeks postoperative follow-up, endoscopic examination showed a viable FAMM flap completely covering the defects (-Fig. 6). The patient later received adjuvant radiation therapy by means of $60 \mathrm{~Gy}$ in 30 fractions. He fully recovered from the diplopia and was temporarily dependent on gastrostomy tube for feeding. At 49 months of follow-up, the flap and its attached masseteric island have now mucosalized and are well perfused with no signs of retraction ( - Fig. 7). The most recent magnetic resonance imaging (MRI) showed stability in residual disease at the clivus and retropharyngeal space (-Fig. 8 ).

\section{Conclusion}

Although successful reconstruction was achieved in our patient, the retrograde FAMM flap should only be used as an alternative when other clinically proven options are not suitable. This article serves as an opener to newer indications of the FAMM flap in head and neck reconstruction of skull base defects.

\section{Financial Disclosure \\ None.}

Conflict of Interest

None.

\section{References}

1 Pribaz J, Stephens W, Crespo L, Gifford G. A new intraoral flap: facial artery musculomucosal (FAMM) flap. Plast Reconstr Surg 1992;90(3):421-429

2 Ayad T, Kolb F, De Monès E, Mamelle G, Tan HK, Temam S. [The musculo-mucosal facial artery flap: harvesting technique and indications]. Ann Chir Plast Esthet 2008;53(6):487-494

3 Dolderer JH, Hussey AJ, Morrison WA. Extension of the facial artery musculomucosal flap to reconstruct a defect of the soft palate. J Plast Surg Hand Surg 2011;45(4-5):208-211

4 Massarelli O, Gobbi R, Raho MT, Tullio A. Three-dimensional primary reconstruction of anterior mouth floor and ventral tongue using the 'trilobed' buccinator myomucosal island flap. Int J Oral Maxillofac Surg 2008;37(10):917-922

5 Xie L, Lavigne F, Rahal A, Moubayed SP, Ayad T. Facial artery musculomucosal flap for reconstruction of skull base defects: a cadaveric study. Laryngoscope 2013;123(8):1854-1861

6 Hadad G, Bassagasteguy L, Carrau RL, et al. A novel reconstructive technique after endoscopic expanded endonasal approaches: vascular pedicle nasoseptal flap. Laryngoscope 2006;116(10): $1882-1886$ 